\title{
Heat kernels and thermodynamics in Rindler space
}

\author{
R. Emparan* \\ Departamento de Física de la Materia Condensada, Universidad del País Vasco, Apartado 644, 48080 Bilbao, Spain
}

(Received 13 July 1994)

\begin{abstract}
We point out that using the heat kernel on a cone to compute the first quantum correction to the entropy of Rindler space does not yield the correct temperature dependence. In order to obtain the physics at arbitrary temperature one must compute the heat kernel in a geometry with different topology (without a conical singularity). This is done in two ways, which are shown to agree with computations performed by other methods. Also, we discuss the ambiguities in the regularization procedure and their physical consequences.
\end{abstract}

PACS number(s): 04.62.+v, 05.70.Ce, 11.10.Wx

The behavior of quantum fields in black hole backgrounds has been actively investigated for the last 20 years [1]. Thus it may seem surprising that computations of thermodynamical magnitudes such as the entropy associated with quantum fields in such geometries have been carried out only recently. It was not until the pioneering work of ' $t$ Hooft [2] that the fundamental relevance of the divergence of these quantities has been fully appreciated. In recent months there has been a considerable amount of work analyzing thermodynamical magnitudes in spaces with horizons. Much of this work has been carried out in Rindler space, i.e., the space of uniformly accelerated observers. It shares most of the qualitative features of black holes and is simple enough to allow a detailed analysis. In particular, the Hawking temperature follows immediately from the geometry of $D$-dimensional Euclidean Rindler space:

$$
d s^{2}=\xi^{2} d \theta^{2}+d \xi^{2}+\sum_{i=1}^{N} d y_{i}^{2}
$$

Here $y_{i}$ are the $N=D-2$ transverse flat coordinates, $\theta$ is the analytically continued Euclidean time, and lines $\xi=$ const correspond to uniformly accelerated observers. The structure of this manifold is $C_{\beta} \times M_{D-2}$, where $M_{D-2}$ is the transverse flat space and $C_{\beta}$ is a flat cone with angular periodicity $\theta \sim \theta+\beta$. Therefore, to avoid the conical singularity at the horizon $(\xi=0), \theta$ must have period $\beta_{H}=2 \pi$.

Several methods have been employed to calculate quantum corrections to the thermodynamical entropy of Rindler space. In [3-5] the statistical mechanics mode counting is carried out after obtaining the energy spectrum of a scalar field in Rindler space in the WKB approximation. Since the density of levels diverges due to the infinite shift of frequencies near the horizon, a cutoff $\xi_{\min }=\epsilon$ must be introduced. The resulting free energy, for the massless case in four dimensions, is

*Electronic address: wmbemgar@lg.ehu.es

$$
F(\beta)=-\frac{\pi^{2} A}{180 \epsilon^{2} \beta^{4}}
$$

and shows the characteristic proportionality to the area. An independent calculation of Dowker makes use of early determinations of the finite temperature stress-energy tensor in Rindler space [6] and obtains exactly this same result [7]. Temperature enters here by letting the period $\beta$ take arbitrary values, and the stress-energy tensor is obtained from the Green function on $C_{\beta} \times M_{D-2}$.

The nontrivial topological structure of Rindler space was exploited by Callan and Wilczek in a variety of ways [8], and allowed them to develop powerful methods to compute the entropy. Calculations were performed mostly in two dimensions, where the divergence is logarithmic and coefficients are universal (cutoff independent). To go to higher dimensions they proposed to employ the heat kernel of the Laplacian on $C_{\beta} \times M_{D-2}$, defined as

$$
\zeta(\tau)=\operatorname{tr} e^{\tau\left(\Delta-m^{2}\right)},
$$

from where the free energy could be obtained:

$$
F(\beta)=-\frac{1}{2 \beta} \int_{0}^{\infty} \frac{d \tau}{\tau} \zeta(\tau) .
$$

Then the entropy follows from the standard thermodynamical formula

$$
S(\beta)=\left(\beta \frac{\partial}{\partial \beta}-1\right) \beta F(\beta) .
$$

Now, in a product space the heat kernel of the Laplacian decomposes into factors corresponding to each subspace: from $M_{D-2}$ we obtain $V_{D-2} /(4 \pi \tau)^{\frac{D-2}{2}}$, whereas, on the cone $C_{\beta}[9]$,

$$
\begin{aligned}
\zeta_{\beta}(\tau) & =\frac{1}{4 \pi \tau}\left\{\frac{\beta}{2 \pi} \pi R^{2}+\tau \frac{\beta}{6}\left[\left(\frac{2 \pi}{\beta}\right)^{2}-1\right]+\cdots\right\} \\
& =(\text { terms linear in } \beta)+\frac{\pi}{6 \beta}+\cdots
\end{aligned}
$$

( $R$ is a cutoff for large radius $\xi$, and the dots indicate terms that vanish for $R \rightarrow \infty$ ). When computing the 
free energy, terms linear in $\beta$ disappear after subtraction of the $\beta=\infty$ contribution [7] [they are automatically eliminated from the entropy by the prescription (5)]. The lower integration limit in Eq. (4) needs short distance regularization $\tau>\epsilon^{2}$. Then, for general $D>2(N>0)$, and $m^{2}=0$, one finds

$$
F(\beta) \propto \frac{V_{N}}{\beta^{2} \epsilon^{N}} .
$$

We see that this procedure yields the correct $V_{N}$ proportionality of the entropy and the divergence $\epsilon^{-N}$. However, if we compare Eq. (7) (for $N=2$ ) with Eq. (2) we notice the different dependence on $\beta$. Although the powerlike dependence on the regulator $\epsilon$ precludes direct comparison of numerical coefficients, the temperature behavior has a physical significance. The introduction of temperature by using geometrical concepts may seem obscure, but the sum over states in statistical mechanics has a clear physical meaning. In the following we shall construct the heat kernel that yields thermal magnitudes at arbitrary temperature.

Rindler space can be regarded as a redefinition of Minkowski space, but their physical properties differ due to the different choice of timelike Killing vectors for each space. This leads to a different definition of positive frequency states, and therefore, different vacua and Feynman propagators. As regards to thermodynamics, the Hamiltonians in the partition function $Z(\beta)=$ $\operatorname{tr} \exp (-\beta H)$ are different in each case. We shall motivate the construction of the heat kernel starting from the expression of the partition function as a path integral in Euclidean field theory. As pointed out by Barbón in Ref. [10], since the action is the same in the inertial and accelerated frames, differences must arise from the choice of the functional integration measure.

The Euclidean action of a massive scalar field in $D$ dimensional Rindler space can be written as

$$
S[\phi]=-\frac{1}{2} \int d \theta d^{N} y d \xi \xi \phi\left(\Delta-m^{2}\right) \phi,
$$

with $\Delta$ the Laplacian in polar coordinates (1). This way of rewriting the action of a scalar in flat space singles out the Euclidean time $\theta$, whose periodicity properties will determine the thermal behavior.

In order to define the appropriate functional integration for Rindler space we find it convenient to introduce the "optical" metric [11]

$$
d \tilde{s}^{2}=\frac{d s^{2}}{g_{00}}=d \theta^{2}+\xi^{-2}\left(d \xi^{2}+\sum_{i=1}^{N} d y_{i}^{2}\right)
$$

$\left(\sqrt{\tilde{g}}=\xi^{-N-1}\right)$ and rescale the field by $\tilde{\phi}=\xi^{N / 2} \phi$. The action is now rewritten as

$$
S[\tilde{\phi}]=-\frac{1}{2} \int d \theta d^{N} y d \xi \sqrt{\tilde{g}} \tilde{\phi}\left(\tilde{\Delta}+\frac{N^{2}}{4}-\xi^{2} m^{2}\right) \tilde{\phi}
$$

$\widetilde{\Delta}$ is the Laplacian in the optical metric (9). An additional term $N^{2} / 4$ has appeared due to the nonzero cur- vature $\tilde{R}=N(N+1)$ of the optical space.

Consider now the partition function over fields periodic under $\theta \sim \theta+k \beta(k \in Z)$ :

$$
Z(\beta)=\int \mathcal{D} \tilde{\phi} e^{-S[\tilde{\phi}]},
$$

with measure $\mathcal{D} \tilde{\phi} \sim \prod_{\theta, \xi, y_{i}} \tilde{g}^{1 / 4} d \tilde{\phi}\left(\theta, \xi, y_{i}\right)$. Functional measures are formally specified by some inner product of functions. Our choice of measure corresponds to the usual inner product in optical space. This is adequate, since the spatial section of this product is the same as the Klein-Gordon product in Rindler space, which, in turn, is determined by the specific choice of timelike Killing vector. Actually, we shall see that this will be enough to obtain the same thermodynamics as the one constructed from Rindler mode counting $[4,5]$.

Our partition function is thus given by

$$
\begin{aligned}
\ln Z(\beta) & =-\frac{1}{2} \operatorname{lndet}\left(-\widetilde{\Delta}-\frac{N^{2}}{4}+\xi^{2} m^{2}\right) \\
& =\frac{1}{2} \int_{0}^{\infty} \frac{d \tau}{\tau} \operatorname{tr} e^{\tau\left(\widetilde{\Delta}+N^{2} / 4-\xi^{2} m^{2}\right)}
\end{aligned}
$$

Therefore the object to be calculated is the heat kernel of the Laplacian in optical space. The optical geometry is quite different from $C_{\beta} \times M_{D-2}$. The structure of (9) is $S^{1} \times \mathcal{H}^{N+1}$, the hyperbolic space $\mathcal{H}^{N+1}$ being the $N+1$ dimensional generalization of the Poincaré upper half plane. Euclidean time has become a coordinate on the circle $S^{1}$. Thus there is no conical singularity in this space. Using the factorization property we see that all the temperature dependence is contained in the heat kernel of the operator $(\partial / \partial \theta)^{2}$ on $S^{1}$. This is a wellknown object. Subtraction of the zero temperature term leaves

$$
\zeta_{S^{1}}(\tau)=\frac{2 \beta}{\sqrt{4 \pi \tau}} \sum_{r=1}^{\infty} e^{-r^{2} \beta^{2} / 4 \tau}
$$

The heat kernel on $\mathcal{H}^{N+1}$ can be computed using the DeWitt-Schwinger expansion

$$
\zeta(\tau)=\frac{1}{(4 \pi \tau)^{\frac{N+1}{2}}} \sum_{k \geq 0}\left[a_{k}\right] \tau^{k} .
$$

The coefficients $\left[a_{k}\right]$ can be expressed in terms of volume integrals of powers and derivatives of the curvature tensor. In particular, $\left[a_{0}\right]$ is given by the volume of $\mathcal{H}^{N+1}$. This is a divergent quantity and needs to be regularized: we restrict the transverse $y_{i}$ space to a box of volume $V_{N}$ and introduce a lower bound $\epsilon$ for $\xi$ :

$$
\left[a_{0}\right]=V_{\mathcal{H}^{N+1}}=\frac{V_{N}}{N \epsilon^{N}}
$$

(for $N=0$ we find the familiar logarithmic divergence).

Higher coefficients are simpler in the massless four dimensional case. In this case $\left[a_{1}\right]$ vanishes, whereas the contribution of $\left[a_{2}\right]$ to $F(\beta)$ is a constant independent 
of the temperature, which can be subtracted out. Regarding higher coefficients, their contribution to $F(\beta)$ vanishes when $\zeta$-function regularization of sums is employed. Therefore, we will discard all these terms. Below we shall find by an independent method that indeed these terms do not appear. Thus, the heat kernel appropriate for Rindler space thermodynamics is

$$
\zeta_{R}(\tau)=\frac{A \beta}{\epsilon^{2}} \frac{1}{(4 \pi \tau)^{2}} \sum_{r \geq 1} e^{-r^{2} \beta^{2} / 4 \tau}
$$

Substitute now Eq. (16) into Eq. (4). The temperature dependence can be easily extracted out by rescaling $\tau \rightarrow$ $\beta^{2} \tau$. The remaining integral and series yield a numerical factor that is easy to compute. Equation (2) is then exactly reproduced.

We see that in this approach the origin of divergences is traced to the infinite volume of optical space. This conclusion was also arrived at by different means in [10].

We now rederive this result following a different approach, which emphasizes other quantum aspects of Rindler space.

Take a basis of eigenfunctions of $-\widetilde{\Delta}-N^{2} / 4+\xi^{2} m^{2}$ :

$$
\psi_{n p \nu}(x)=\frac{\sqrt{2 \nu \sinh \pi \nu}}{(2 \pi)^{\frac{N+1}{2}} \pi} e^{i 2 \pi n \theta / \beta+i p_{i} y_{i}} \xi^{N / 2} K_{i \nu}(\mu \xi) .
$$

We have denoted $x=\left(\theta, \xi, y_{i}\right)$ and $\mu=\sqrt{p^{2}+m^{2}} ; K_{i \nu}$ is the modified Bessel function. The corresponding eigenvalues are $\left(4 \pi^{2} n^{2} / \beta^{2}+\nu^{2}\right)$. The functions (17) are orthonormal with respect to the volume $D$ form of the optical geometry. [The partition function (11) and its integration measure become especially simple when $\tilde{\phi}$ is expanded in this basis.]

As is known, all the relevant information can be encoded in the heat function

$$
K\left(x^{\prime}, x^{\prime \prime} ; \tau\right)=\sum_{n p \nu} e^{-\tau\left(4 \pi^{2} n^{2} / \beta^{2}+\nu^{2}\right)} \psi_{n p \nu}^{*}\left(x^{\prime}\right) \psi_{n p \nu}\left(x^{\prime \prime}\right)
$$

For example, the Euclidean two-point Green function in Rindler space can be obtained as

$$
G_{E}\left(x^{\prime}, x^{\prime \prime}\right)=\left(\xi^{\prime} \xi^{\prime \prime}\right)^{-N / 2} \int_{0}^{\infty} d \tau K\left(x^{\prime}, x^{\prime \prime} ; \tau\right),
$$

which can be readily seen to agree with previous calculations in the literature.

The integrated heat kernel follows from tracing over spacetime indices:

$$
\zeta(\tau)=\int d^{D} x \sqrt{\tilde{g}} K(x, x ; \tau) .
$$

Now it is interesting to see how divergences appear in this approach. The eigenvalue problem in Rindler space is not well defined unless we introduce a cutoff near the horizon. Aside from discretizing the spectrum, this has the effect that the spectrum of frequencies $\nu$ becomes dependent on the transverse momentum $p$ and bounded below by a nonzero value $\nu \geq \epsilon \mu$. Also, inner products of eigenfunctions are now determined by

$$
\int_{\epsilon}^{\infty} \frac{d \xi}{\xi} K_{i \nu}(\mu \xi) K_{i \nu}(\mu \xi)=\frac{\pi^{2}}{2 \nu \sinh \pi \nu} \frac{d n}{d \nu} .
$$

The eigenvalue density $d n / d \nu$ has been computed in [4] using the WKB method, and is seen to diverge for $\epsilon \rightarrow$ 0 . Substitution leads, after Poisson resummation, to the same heat kernel found above.

Some remarks are now in order. Notice that the regularization procedure employed when obtaining Eq. (7), i.e., cutting off short proper times $\tau$, is different from the prescription $\xi_{\min }=\epsilon$ used in Eqs. (2) and (16). In fact, calculations using the Rindler metric (1) admit both regularizations [12], but there does not seem to be any $a$ priori reason to choose one of them. The choice of one scheme or the other must be based on physical grounds, since the physics described in each case turns out to be different. We have pointed out that short proper time regularization yields unphysical temperature dependence. On the other hand, the Euclidean path integral formalism instructs us to perform computations in the optical geometry, where the regularization procedure is automatically selected in much the same way as any infinite volume regularization. We have shown that this is the right way to proceed, and that indeed it yields the correct $\beta$ dependence. Also, it is worth remarking that the optical geometry allows for a physical interpretation in terms of a sum over states through the factor $\zeta_{S^{1}}(\tau)$, Eq. (13).

One last remark. Rindler space can be regarded as the large mass limit of the Schwarzschild black hole. For a finite mass black hole with Euclidean geometry $R^{2} \times S^{D-2}$, the optical rescaling leads to a spacetime with a $S^{1}$ factor. This yields the temperature dependence through the same function as Eq. (13). Computation of the remaining factor becomes more involved, but the qualitative features are not different. Actually, the same arguments can be applied to find that only $\left[a_{0}\right]$ and $\left[a_{1}\right]$ are needed (in four dimensions). Also, the divergences of optical volume that appear in $\left[a_{0}\right]$ are seen to be general.

I would like to thank M. A. Goñi, J. L. Mañes, and M. A. Valle for useful discussions and encouragement. This work has been partially supported by a FPI grant from MEC (Spain) and by projects UPV 063.320-EB119-92 and CICYT AEN93-0435.
[1] N. D. Birrell and P. C. W. Davies, Quantum Fields in Curved Space (Cambridge University Press, Cambridge, England, 1982).

[2] G. 't Hooft, Nucl. Phys. B256, 727 (1985).
[3] H. J. de Vega and N. Sánchez, Nucl. Phys. B299, 818 (1988).

[4] L. Susskind and J. Uglum, Phys. Rev. D 50, 2700 (1994).

[5] D. Kabat and M. J. Strassler, Phys. Lett. B 329, 46 
(1994).

[6] J. S. Dowker, J. Phys. A 10, 115 (1977); Phys. Rev. D 18, 136 (1978).

[7] J. S. Dowker, Class. Quantum Grav. 11, L55-L60 (1994).

[8] C. Callan and F. Wilczek, Phys. Lett. B 333, 55 (1994).

[9] D. V. Fursaev, Class. Quantum Grav. 11, 1431 (1994).

[10] J. L. F. Barbón, Phys. Rev. D 50, 2712 (1994).

[11] G. W. Gibbons and M. J. Perry, Proc. R. Soc. London
Ser. A 358, 467 (1978).

[12] This point has been raised to us by D. Kabat (private communication). One can consider the geometry of (1), but with the apex of the cone excised. Then, taking due care of the boundary at $\xi=\epsilon$, the heat kernel computed in this space yields the free energy Eq. (2). Again, notice that this geometry does not have any conical singularity. 